\title{
Research Article \\ Effect of the Tip Size on AFM Cantilever Based Force Sensor
}

\author{
Yajing Shen \\ Mechanical and Biomedical Engineering Department, City University of Hong Kong, Tat Chee Avenue, Kowloon, Hong Kong \\ Correspondence should be addressed to Yajing Shen; yajishen@cityu.edu.hk
}

Received 31 March 2015; Accepted 27 May 2015

Academic Editor: Giuseppe Ferri

Copyright (C) 2015 Yajing Shen. This is an open access article distributed under the Creative Commons Attribution License, which permits unrestricted use, distribution, and reproduction in any medium, provided the original work is properly cited.

\begin{abstract}
Atomic force microscopy (AFM) cantilever is a widely used end effector for precise force sensing and micro-nanomanipulation at small scale. However, in current researches, the effect of the cantilever tip on the force sensing and manipulation accuracy is rarely considered. In this paper, we investigate how the tip size of the end effector affects the measurement accuracy of the cell adhesion force. First, several end effectors with different tip sizes are fabricated from the same AFM cantilever via focused ion beam (FIB) etching. Then, the single cell detachment force is measured at the same experiment condition by these end effectors, respectively. The results indicate that the sensed adhesion force is susceptible to the tip size of the end effector obviously. In addition, the precision of cell manipulation is also regulated by the contact area between the cell and end effector greatly. These findings will benefit our in-depth understanding on the force interaction at small scale and will provide valid reference for the development of high-precision force sensor and manipulation.
\end{abstract}

\section{Introduction}

AFM cantilever is a commercial end effector for the atomic force microscopy, which is used to image the sample based on the force interaction between the cantilever tip and the sample surface. Nowadays, the AFM cantilever has been taken as a powerful micro-nanoforce sensor owing to its high force sensing resolution and widely sensing range [14]. In addition, AFM cantilever is also a powerful tool for the mechanical property characterization of micro-nanomaterials after it is integrated within the scanning electron microscopy (SEM) [5-7].

The adhesion force between biological cell and extracellular matrix is extremely important in biology field, since it dominates many biological activities, such as cell's viability, morphogenesis, and orientation $[8,9]$. Nowadays, AFM cantilever has been successfully used to study the cell-substrate adhesion strength, which has the advantage of tiny size, high force resolution, high usability, and so on [10-12]. Here, AFM cantilever is driven to push and detach the cell from the substrate, during which process the maximum adhesion strength is calculated based on the deflection of AFM cantilever beam. This measurement approach can provide high force sensing resolution owing to the working principle of the AFM cantilever. However, the AFM cantilever is usually with an ultrasharp tip approximately several nanometers. The cell wall/membrane may be damaged due to the extremely small contact area if the sharp tip is directly used to detach the cell from substrate.

To reduce the physical damage to the cell, scientists proposed to use the backside of the cantilever or use the milled cantilever tip to push the cell, by which a larger contact area can be obtained [13-17]. However, the force sensing principle of the AFM cantilever is based on the deflection of the cantilever beam. When the tip size of the end effector is too large, the cell would be subjected to an unexpected pressing force owing to the cantilever deflection, which would result in sensing error to the force measurement. In addition, the surface force becomes dominant at small scale [18], which raises difficulties to the accurate cell manipulation, since the cell always trends to adhere to the surface of the end effector.

Force sensing and sample manipulation are two significant functions of the AFM cantilever based end effector. It has been believed that the accuracy of sensing and manipulation relates to the end effector itself somehow, but the behind mechanism is still unknown at small scale. Specifically, few literatures have been reported to discuss the size effect of the end effector's tip on the force sensing and cell manipulation. 


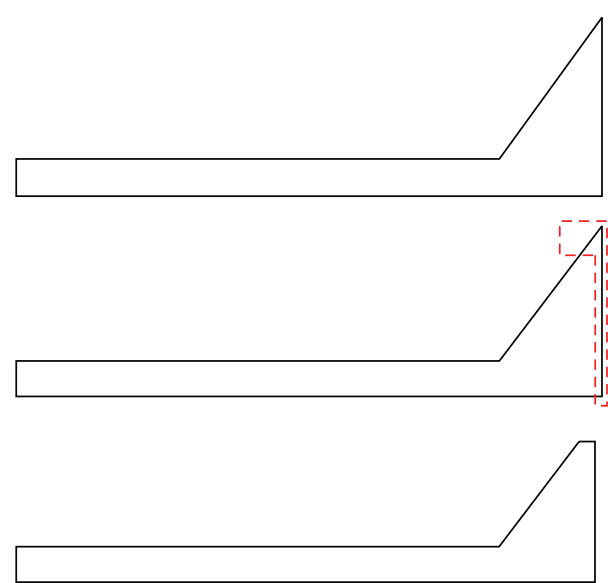

(a)

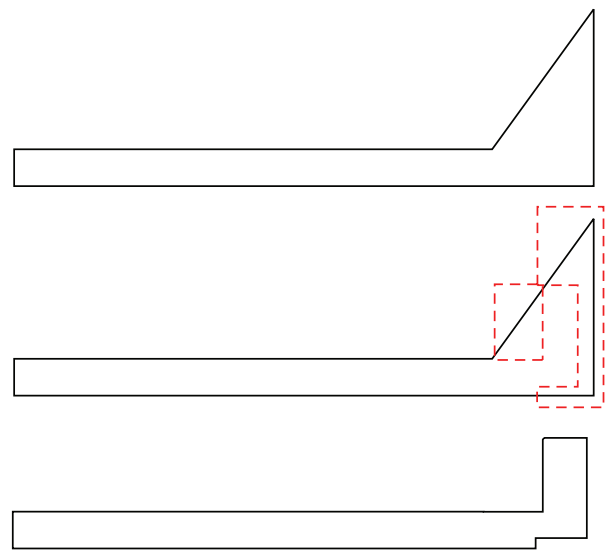

(c)

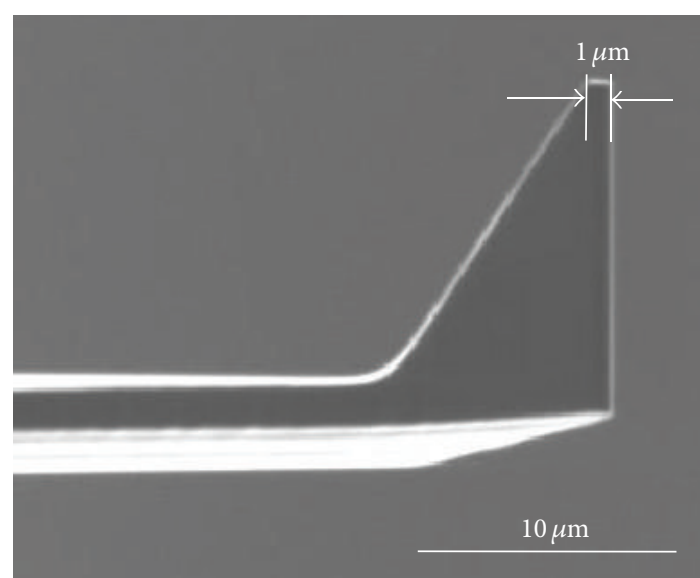

(b)

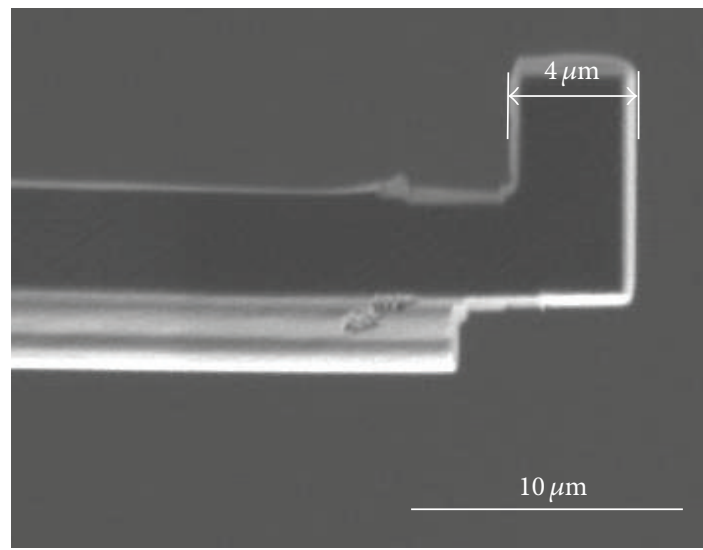

(d)

Figure 1: Preparation of the two end effectors with different tip sizes. (a) and (b) Fabrication of the end effector with small tip size. (c) and (d) Fabrication of the end effector with large tip size.

In this paper, we investigate the effect of the end effector's tip size on the force sensing and cell manipulation in the cell detachment process. First, three end effectors with different tip sizes are fabricated from AFM cantilever based on focused ion beam (FIB) etching. Then, they are used to detach the cell from the substrate and the force is calculated from the deflection of the cantilever beam. After that, the effect of the end effector's size on the force sensing accuracy is discussed. Finally, two application cases are demonstrated to illustrate how the size of the end effector affects cell manipulation.

\section{Materials and Methods}

2.1. Preparation of the End Effector from AFM Cantilever. The two end effectors were fabricated from the same type of AFM cantilever (OMCL-AC240TM, Olympus Inc.) by FIB etching technique. Here, the AFM cantilever was put inside the chamber of FIB first. Then, the gallium primary ion $\left(\mathrm{Ga}^{+}\right)$ was accelerated to hit the tip of the AFM cantilever, thereby to remove the material gradually. Finally, the sharp tip was able to be milled to a flat plane. Figure 1(a) shows the fabrication process of the end effector with small tip size. The rectangles indicate the area removed by FIB etching. Figure 1(b) gives the SEM image of the fabricated end effector, the tip size of which is around $1.0 \mu \mathrm{m}(\mathrm{H}) \times 2.0 \mu \mathrm{m}(\mathrm{W})$. As shown in Figure $1(\mathrm{c})$, the end effector with a larger tip size was also fabricated through the similar FIB etching process. The image of this end effector is shown in Figure 1(d), the tip size of which is around $4.0 \mu \mathrm{m}(\mathrm{H}) \times 2.0 \mu \mathrm{m}(\mathrm{W})$.

The two end effectors have the same spring constant $2.4 \mathrm{~N} / \mathrm{m}$ and the same beam length $240 \mu \mathrm{m}$, since they are fabricated from the same type of AFM cantilever. Therefore, they would have the same deflection when the same force is applied on their tips. In this paper, these two end effectors will be employed to measure the cell adhesion strength.

2.2. Preparation of Yeast Cell. Yeast is classified in the fungi. It is one of the oldest biological cells that are used in our life, such as those for the bread and beer making. Nowadays, yeast cell has also been taken as the model to study human organism owing to its relative simple structure. In this paper, yeast cell (W303) is taken as the biosample, the diameter of which is approximately $3-6 \mu \mathrm{m}$. First, yeast cells were cultured on YPD plate for 48 hours at $30^{\circ} \mathrm{C}$ incubator. Then, 
yeast clones were picked up by tooth picker and dissolved in pure water. After that, $2 \mu \mathrm{L}$ biosolution with yeast cells was dropped on the substrate surface. After water evaporation, the yeast cells adhered to the substrate surface, which would be used for the following detachment study.

2.3. Experimental Setup for Cell Adhesion Measurement. Environmental scanning electron microscope (ESEM) is a unique type of SEM. It allows the observation of water contained sample in real time with nanometer resolution, which provides an effective approach for the biological sample study. In this paper, the substrate with cells is put inside the ESEM chamber. At the ESEM mode, the relative humidity around the cell can be controlled up to $100 \%$, so the yeast cell can maintain its nature condition without shrinking or cracking.

Previously, we have developed a nanorobotic manipulation system for the ESEM chamber. It has seven degrees of freedom (DOFs) in total and is able to provide a positioning resolution approximately $30 \mathrm{~nm}$. Here, the end effector is assembled on the nanorobotic manipulation system, by which the position of the end effector can be precisely controlled. More detailed specifications about the nanorobotic manipulation system and the ESEM can be found from elsewhere [19].

A schematic drawing of the single cell adhesion strength measurement is illustrated in Figure 2. First, the end effector is assembled on the nanorobotic manipulator inside ESEM chamber. Then, driven by the nanorobotic manipulator, the end effector moves towards, touches, and detaches the yeast cell from the substrate gradually. Since the end effectors are fabricated from AFM cantilever, the value of adhesion strength $F$ can be calculated by the Hooke's law $F=k \delta$, where $k$ is the spring constant of the cantilever and $\delta$ is the maximum deflection of the cantilever beam at the tip point.

In this paper, the spring constant $k$ of the two end effectors is $2.4 \mathrm{~N} / \mathrm{m}$ according to the product manual. The deflection of the cantilever beam $\delta$ is measured from the ESEM images directly by image analysis software (ImageJ, National Institute of Health).

\section{Results and Discussion}

3.1. Adhesion Force Measurement Result. The adhesion strength of yeast cell to substrate is measured by each end effector at the same experiment condition. The images of cell and end effector are observed by SEM in real time during the overall detachment process. Figure 3(a) shows the experiment images of using the end effector with the small tip $(1.0 \mu \mathrm{m})$, and Figure $3(\mathrm{~b})$ shows the images during the cell adhesion measurement process by the end effector with the large tip size $(4.0 \mu \mathrm{m})$. It can be seen clearly that the beam of the end effector with a lager tip has a larger beam deflection in the experiment, which means a larger force is required to detach the cell. In other words, a larger adhesion force is sensed by the measurement system when using the end effector with a larger tip.

Five independent cell detachment experiments are performed at the same experiment condition by each end

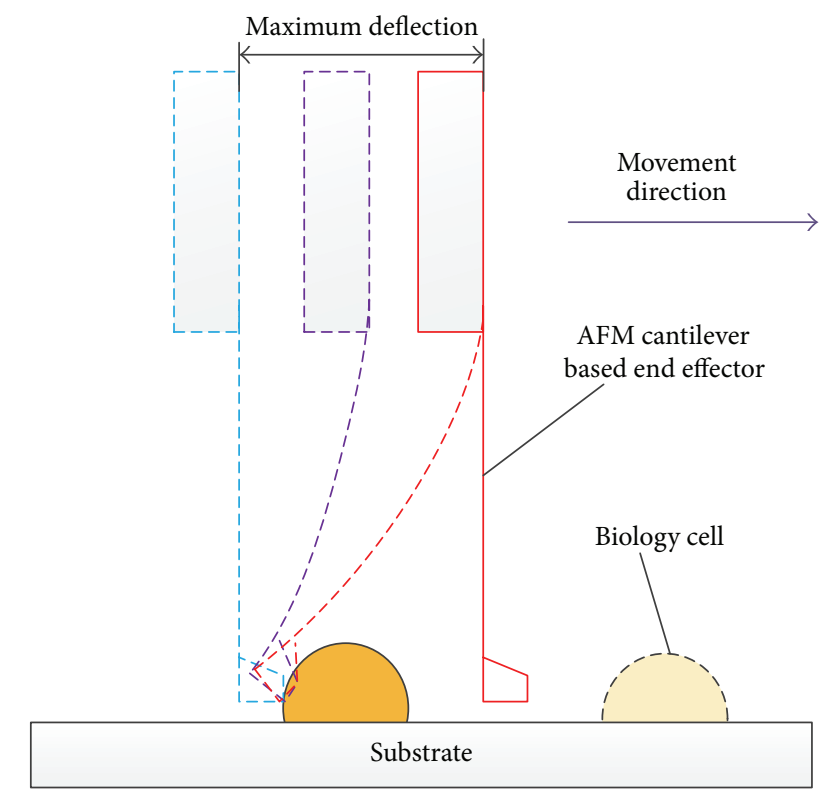

FIGURE 2: Schematics for the cell adhesion force measurement by AFM cantilever based end effector.

TABLE 1: The measured adhesion force by the end effectors with different tip sizes.

\begin{tabular}{lccc}
\hline Measured adhesion force & \multicolumn{3}{c}{ Tip size $(\mu \mathrm{m})$} \\
& 1.0 & 2.5 & 4.0 \\
\hline Average $(\mu \mathrm{N})$ & 24.8 & 35.0 & 65.6 \\
Deviation $(\mu \mathrm{N})$ & 6.4 & 11.7 & 13.1 \\
\hline
\end{tabular}

effector. For comparison, we also measured the adhesion force by end effector with a middle tip size $(H=2.5 \mu \mathrm{m})$, and the statistics adhesion force data are shown in Figure 3(c) and listed Table 1 . The obtained mean adhesion values are $24 \mu \mathrm{N}$, $35 \mu \mathrm{N}$, and $66 \mu \mathrm{N}$ for the end effectors with small, middle, and large tips, respectively. The results clearly show that the sensed force is much more smaller by the end effector with small tip. For example, the force $24 \mu \mathrm{N}$ measured by the end effector with small tip $(1.5 \mu \mathrm{m})$ is less than half of the force $66 \mu \mathrm{N}$ by the end effector with large tip $(4 \mu \mathrm{m})$.

3.2. Effect of Tip Size on Force Sensing Accuracy. The experiment results indicate that the measured adhesion force has a large difference by different end effectors; that is, a larger adhesion force is gained by the end effector with larger tip size. We think one main cause of this difference is from the deflection of the AFM cantilever beam.

As illustrated in Figure 4, the cantilever beam would have a deflection during the cell detachment process due to force sensing principle of AFM cantilever. Therefore, the normal direction of the contact area between the tip and cell surface cannot be exactly vertical to the substrate.

For the end effector with small tip, the pressing force to the cell toward the substrate is not obvious, since this force is dissipated by the local deformation of cell. Thus, the applied 

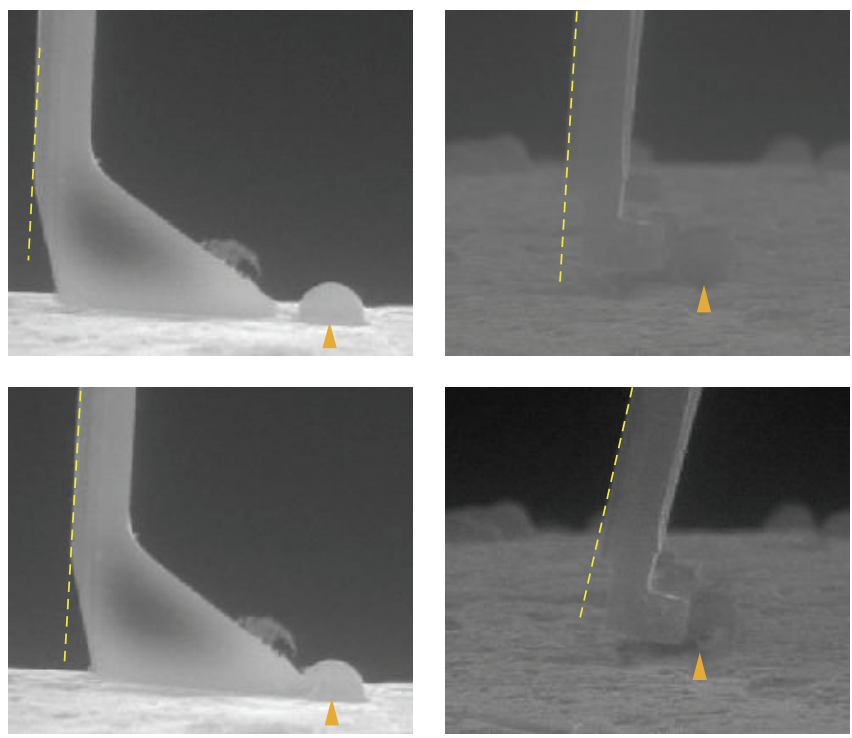

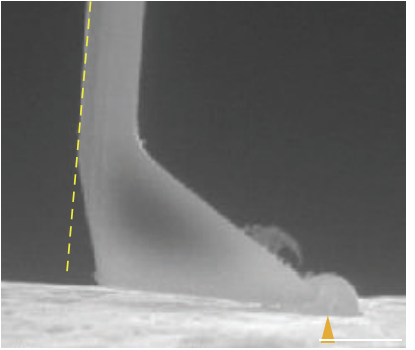

(a)

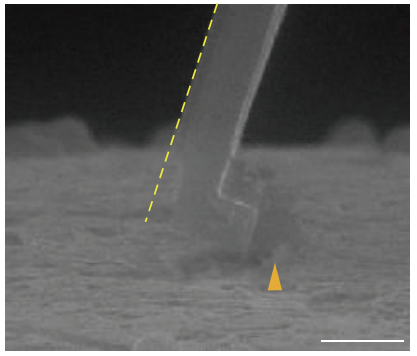

(b)

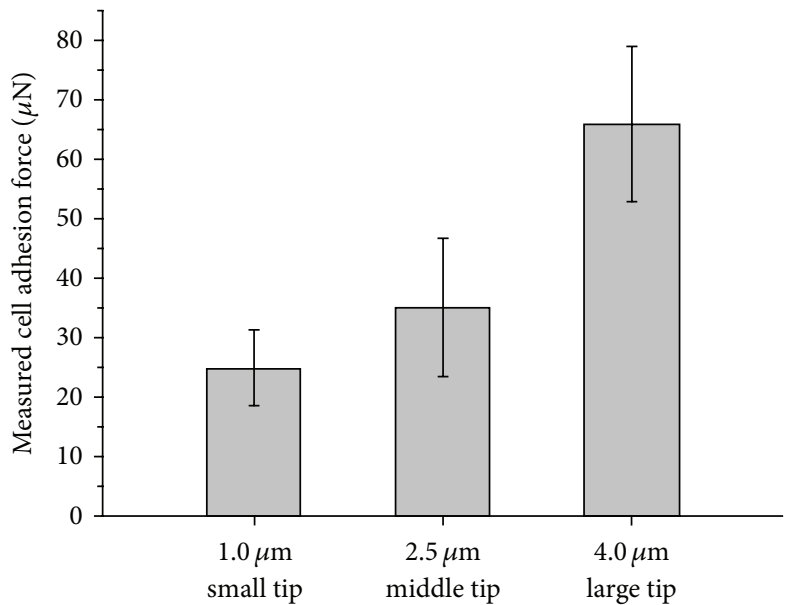

(c)

FIGURE 3: Cell adhesion force measurement results. (a) SEM images of the cell and end effector with small tip size (1.0 $\mu \mathrm{m})$. (b) SEM images of the cell and end effector with large tip size $(4.0 \mu \mathrm{m})$. (c) Comparison of the cell adhesion force by the end effector based on the beam deflection. The scale bar is $5 \mu \mathrm{m}$. The dashing lines indicate the deflection of the cantilever beam and the triangles indicate the position of the cell.

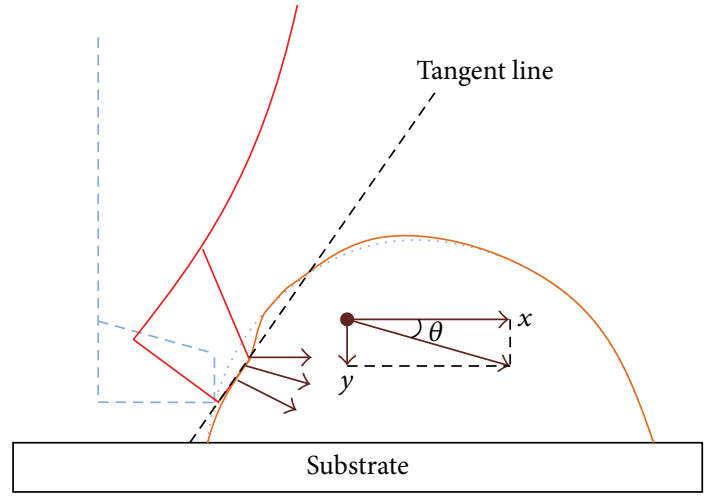

(a)

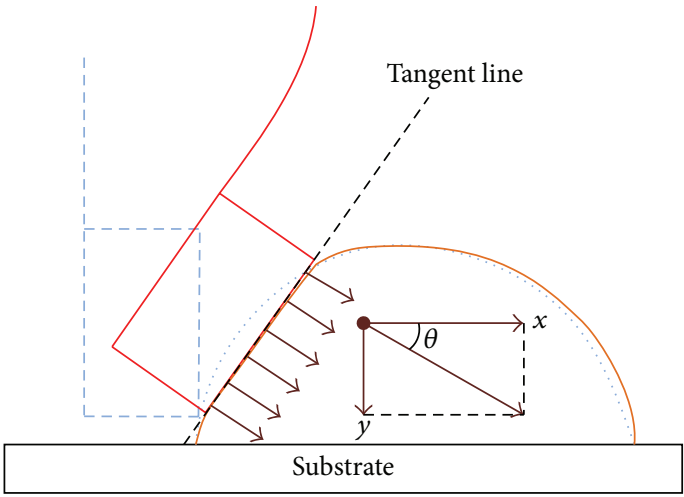

(b)

FIGURE 4: Schematics of the force components caused by the deflection of the AFM cantilever. The calculated force by the cantilever's beam deflection can be divided into two force components: one is to push the cell and the other one is to press the cell. (a) End effector with small tip. (b) End effector with large tip. 


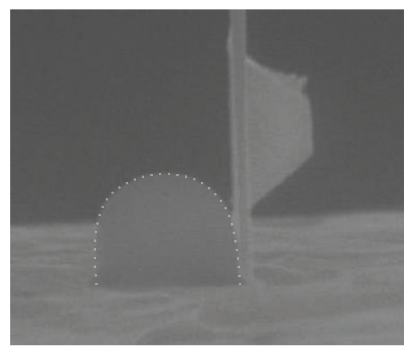

(a1)

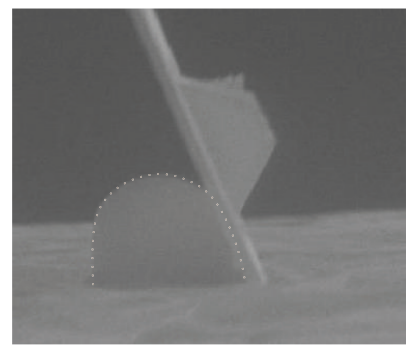

(a5)

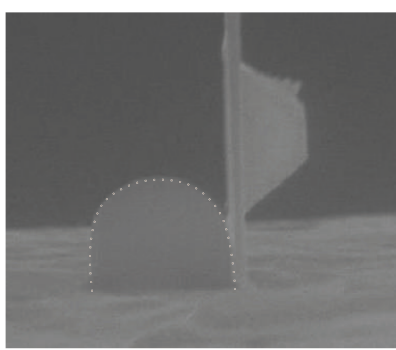

(a2)

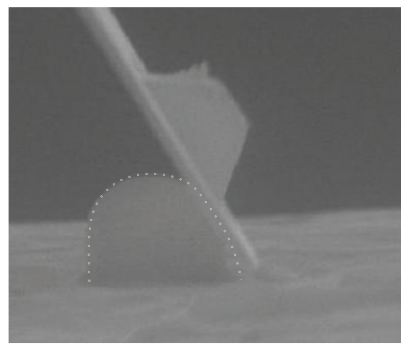

(a6)

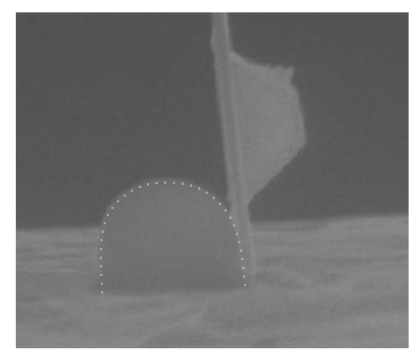

(a3)

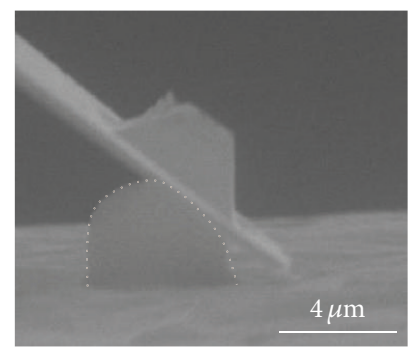

(a7)

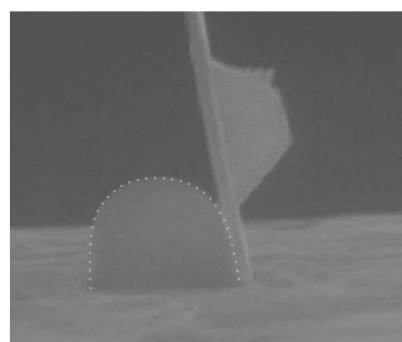

(a4)

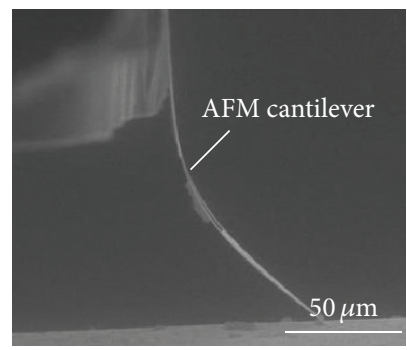

(b)

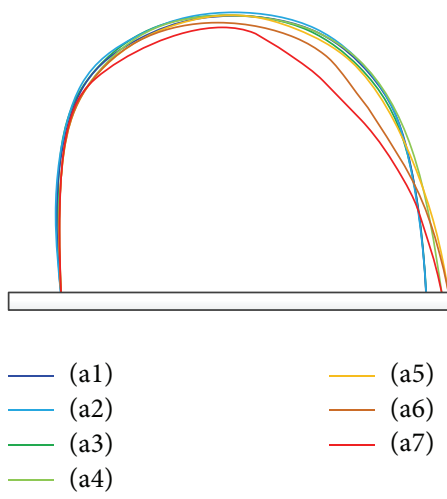

(c)

FIGURE 5: Cell detachment demonstration by the backside of AFM cantilever. (a1-a7) SEM images of the cell detachment process. (b) Deformation of the AFM cantilever beam, corresponding to (a7). (c) Deformation of the cell in the detachment process. The cell is pressed rather than moved on the substrate.

force on the cell is almost along $x$ direction (parallel to the substrate) during the cell detachment process.

On the other hand, for the end effector with large tip, the detachment force divides into two orientations: one is along $x$ direction and the other one is vertically to the substrate. Here, the force along $x$ direction contributes to the cell detachment, and the vertical force presses the cell to the substrate. When the contact area is large, the whole body of the cell is pressed rather than a local deformation. Therefore, the vertical force (press force) is nonignorable in this case. As a result, the measured force from the AFM cantilever, that is, the resultant force, cannot well reflect the detachment force (along $x$ direction). Moreover, the adhesion between the cell and substrate is also enhanced due to the cell press. Therefore, the measured force by the end effector with large tip size is much larger than that by the end effector with small tip size.

To verify the above conclusion, we then used the backside of the AFM cantilever (OMCL-TC800PB, Olympus Inc.) to perform the cell detachment experiment for comparison. As the images shown in Figure 5, the AFM cantilever was fully contacted with the cell owing to its big size. Although the cantilever had have a large deflection (Figures 5(a7) and $5(\mathrm{~b}))$, the force was about $65 \mu \mathrm{N}$, the cell still adhered to the substrate strongly. The deformation information of cell (Figure 5(c)) proves that the cell is pressed rather than detached. This result coincides well with our conclusion that only a few force component contributes to the cell detachment when the beam has a large deflection, especially when the tip size is large.

\subsection{Cell Manipulation Demonstration by End Effectors with} Different Tip Sizes. At small scale, the surface force becomes dominant, which causes difficulties to the accuracy manipulation of sample. Nowadays, many different types of end effectors have been proposed to address the cell manipulation task at small scale [20-22]. However, the effect of the 

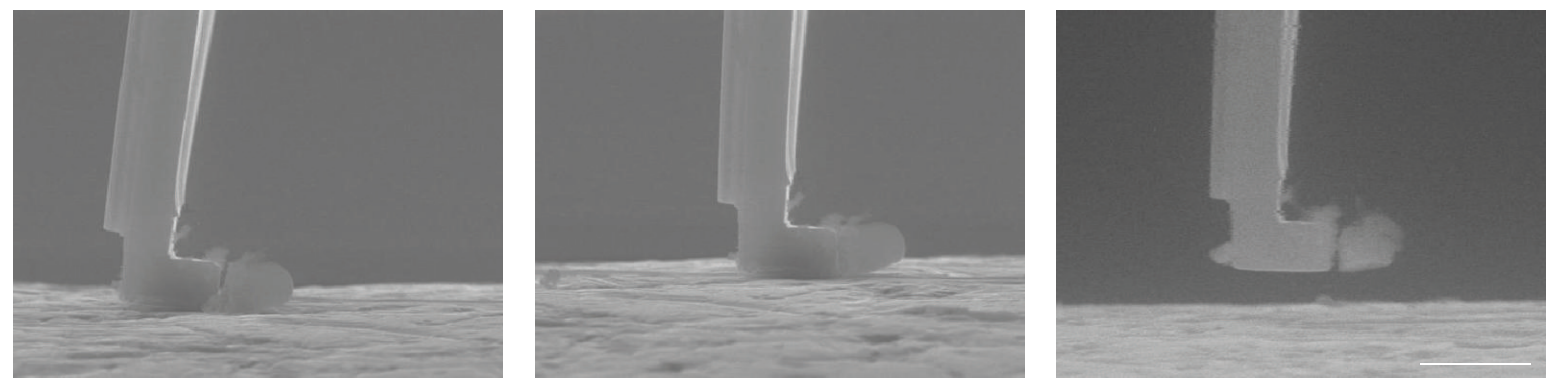

(a)

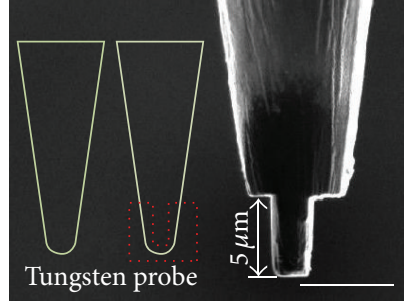

(b)
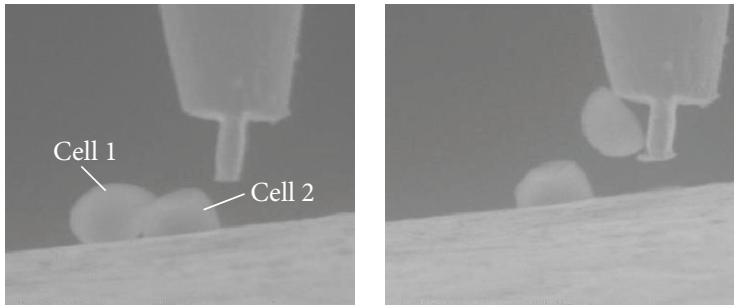

(c)

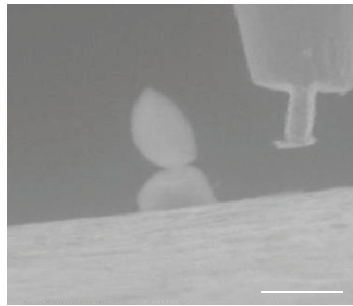

FIGURE 6: Cell transfer demonstration by end effector with large tip size. (a) AFM cantilever based end effector. (b) Tungsten probe based end effector. (c) Cell transfer demonstration.

end effector's size is rarely considered in the manipulation process. Our results have elucidated that the contact area affects the force sensing accuracy. In this section, we will discuss how the cell manipulation is affected by the tip size of the end effector.

3.3.1. Cell Transfer by End Effector with Large Tip Size. As shown in Figure 6(a), after the cell was detached from its initial position, we used the AFM cantilever based end effector (large tip) to push the cell from left to right. Interestingly, the cell was not pushed forward but adhered to the surface of the end effector. After the end effector was moved up, the cell was also lifted up. It indicates that the surface force between the end effector and cell greatly regulates the cell manipulation procedure. To further investigate the effect of surface area on cell manipulation, we designed another end effector with a large tip from a tungsten probe by FIB etching (Figure 6(b)). This tip size $(5 \mu \mathrm{m}(\mathrm{H}) \times 4 \mu \mathrm{m}(\mathrm{W}))$ of this end effector is larger than the size of cell, and thereby it can fully contact with the cell. This end effector was used to push the cell under the driving of the nanorobotic manipulation system. After the cell was detached from its initial position, the cell adhered to the surface of the end effector as expected (Figure 6(c)). Then, the cell was picked up, transferred, and put on the top of another cell successfully.

The above two demonstrations clearly prove that the surface force generated by the end effector with large tip size is nonignorable at small scale. This force not only increases difficulties to the precise cell manipulation, but also makes the force sensing of the manipulation become impossible. However, on the other hand, it provides possibility for the other types of cell manipulation if we can take advantage of this surface force properly, as the cell transfer mentioned above.
3.3.2. Cell Pushing by End Effector with Small Tip Size. For comparison, we performed the similar cell pushing experiment by the end effector with small tip size, and the experimental images are given in Figure 7. The results show that the cell slipped on the substrate gradually under the pushing of the end effector. It means the surface force is not large enough to pick up the cell in this case owing to the small contact area. This result proves that the end effector with small tip size is more suitable for the precise cell manipulation owing to the smaller distraction of the surface force.

3.4. Discussion. AFM cantilever based end effector is widely used as the force sensor or manipulator at micro-nanoscale. The force sensing principle of the AFM cantilever is based on the deflection of the cantilever beam. In the cell adhesion force study, the cell is detached from the substrate under the pushing of the end effector's tip. During this experiment, an avoidable pressing force will be exerted onto the cell due to the deflection of the cantilever beam. This force becomes nonignorable when the contact area between the end effector and the cell is large. Our experiment results indicate that the tip size of the end effector has a great effect on the force sensing accuracy and that the sensed force is larger when using the end effector with large tip. We think this larger force is a measurement error caused by the large deformation of the cantilever beam, especially when the end effector has a large tip. This finding provides guidelines for the development of the AFM cantilever based force sensor, that is, trying to avoid the large deformation and the large contact area.

At small scale, the surface tension forces, electrostatic forces, and van der Waals forces become dominant, which raise new challenges to the precise cell manipulation. Thus, the contact area between the end effector and the sample becomes an import factor regulating the cell manipulation. 


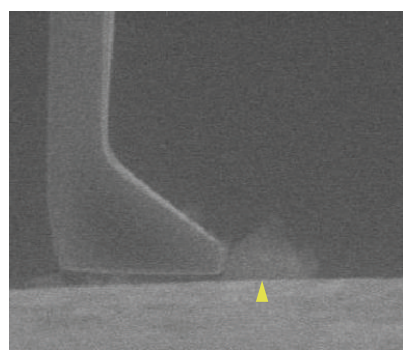

(a)

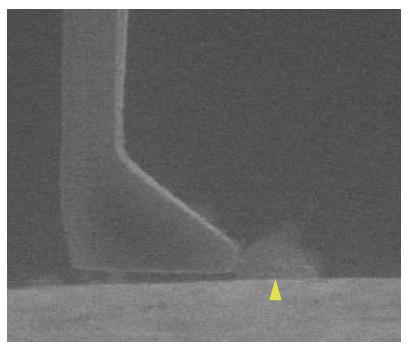

(e)

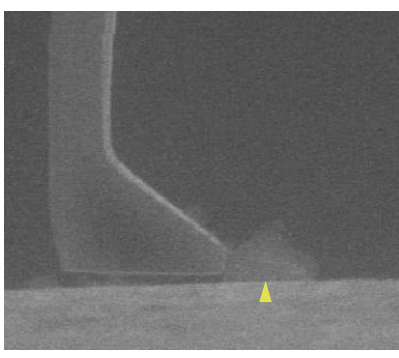

(b)

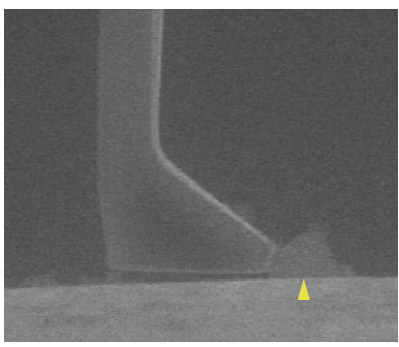

(f)

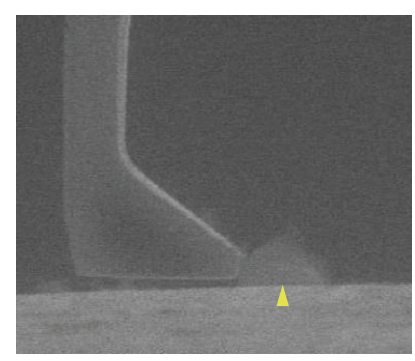

(c)

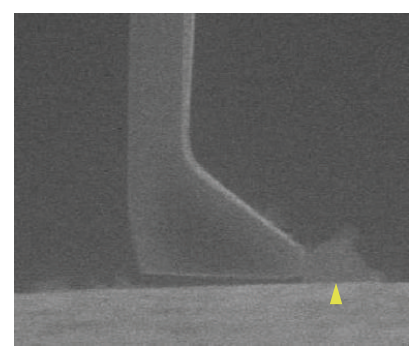

(g)

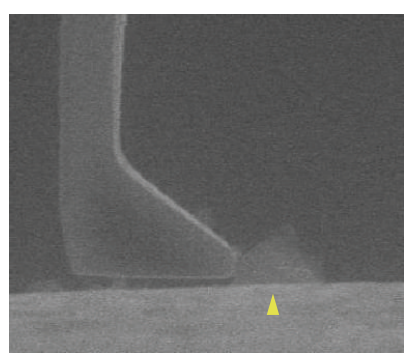

(d)

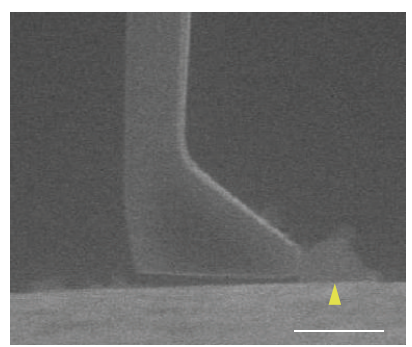

(h)

FIGURE 7: Cell pushing by end effector with small tip size. The cell is pushed to slip on the substrate surface. The scale bar is $5 \mu \mathrm{m}$.

Our results show that the cell would adhere to the end effector surface due to the surface force when using the end effector with large tip. This force not only affects the cell positioning accuracy, but also affects the force sensing ability of the end effector. Therefore, we should avoid using the end effector with large tip size to manipulate the small object.

The results in this paper provide direct evidences to elucidate how the end effector's tip size affects the force sensing and manipulation accuracy at small scale. Generally, a softer AFM cantilever has a higher force resolution. However, our results prove that we should avoid the large deflection of the AFM cantilever when using it as a force sensor. Therefore, the AFM cantilever with suitable spring constant and more importantly with smaller tip should be chosen for the force sensing. In addition, for the precise manipulation purpose, we should also choose a smaller tip to prevent the force disturbance from the unexpected surface force. These findings are important for the in-depth understanding of the surface force interaction and will greatly benefit the development of micro-nanoforce sensor and the research on precise micromanipulation at small scale.

\section{Conclusions}

In this paper, we study the effect of the end effector size on the force sensing and manipulation accuracy at small scale. Two end effectors with different tip sizes are fabricated from the commercial AFM cantilever by FIB etching. Then, the end effector is mounted on the nanorobotics manipulation system within ESEM chamber. The adhesion strength of yeast cell to substrate is measured by the two end effectors, respectively. It is found that the size of the contact area between the end effector and cell affects the force sensing result greatly. Moreover, the contact area also regulates the cell manipulation; that is, a large contact area will generate a nonignorable surface force to adhere to the cell. These findings provide clear evidence that the effect of the tip size on force sensing and micromanipulation will benefit our in-depth understanding of the force interaction at small scale and the development of micro-nanoforce sensor and manipulator.

\section{Conflict of Interests}

The author declares that there is no conflict of interests regarding the publication of this paper.

\section{Acknowledgments}

This research is supported by the National Natural Science Foundation of China (61403323) and the Early Career Scheme from UGC of Hong Kong (9048004).

\section{References}

[1] D. J. Müller and Y. F. Dufrêne, "Atomic force microscopy: a nanoscopic window on the cell surface," Trends in Cell Biology, vol. 21, no. 8, pp. 461-469, 2011.

[2] M. Li, L. Liu, N. Xi et al., "Atomic force microscopy imaging and mechanical properties measurement of red blood cells and aggressive cancer cells," Science China Life Sciences, vol. 55, no. 11, pp. 968-973, 2012.

[3] J. Friedrichs, J. Helenius, and D. J. Muller, "Quantifying cellular adhesion to extracellular matrix components by single-cell force spectroscopy," Nature Protocols, vol. 5, no. 7, pp. 1353-1361, 2010.

[4] A. Zdunek and A. Kurenda, "Determination of the elastic properties of tomato fruit cells with an atomic force microscope," Sensors, vol. 13, no. 9, pp. 12175-12191, 2013.

[5] I. Jimenez-Palomar, A. Shipov, R. Shahar, and A. H. Barber, "Influence of SEM vacuum on bone micromechanics using in 
situ AFM," Journal of the Mechanical Behavior of Biomedical Materials, vol. 5, no. 1, pp. 149-155, 2012.

[6] U. Mick, V. Eichhorn, T. Wortmann, C. Diederichs, and S. Fatikow, "Combined nanorobotic AFM/SEM system as novel toolbox for automated hybrid analysis and manipulation of nanoscale objects," in Proceedings of the IEEE International Conference on Robotics and Automation (ICRA '10), pp. 40884093, IEEE, May 2010.

[7] E. P. S. Tan and C. T. Lim, "Mechanical characterization of nanofibers-a review," Composites Science and Technology, vol. 66, no. 9, pp. 1102-1111, 2006.

[8] M. H. Zaman, L. M. Trapani, A. Siemeski et al., "Migration of tumor cells in 3D matrices is governed by matrix stiffness along with cell-matrix adhesion and proteolysis," Proceedings of the National Academy of Sciences of the United States of America, vol. 103, no. 29, pp. 10889-10894, 2006.

[9] Y. Shen, M. Nakajima, S. Kojima, M. Homma, M. Kojima, and T. Fukuda, "Single cell adhesion force measurement for cell viability identification using an AFM cantilever-based micro putter," Measurement Science and Technology, vol. 22, no. 11, Article ID 115802, 2011.

[10] C. Selhuber-Unkel, T. Erdmann, M. López-García, H. Kessler, U. S. Schwarz, and J. P. Spatz, "Cell adhesion strength is controlled by intermolecular spacing of adhesion receptors," Biophysical Journal, vol. 98, no. 4, pp. 543-551, 2010.

[11] A. M. Baró and R. G. Reifenberger, Atomic Force Microscopy in Liquid: Biological Applications, John Wiley \& Sons, New York, NY, USA, 2012.

[12] A. Yamamoto, S. Mishima, N. Maruyama, and M. Sumita, "A new technique for direct measurement of the shear force necessary to detach a cell from a material," Biomaterials, vol. 19, no. 7-9, pp. 871-879, 1998.

[13] N. Piacentini, A. B. Verkhovsky, C. Gabella, J.-J. Meister, and B. Vianay, "Ultra-soft cantilevers and 3-d micro-patterned substrates for contractile bundle tension measurement in living cells," Lab on a Chip, vol. 14, no. 14, pp. 2539-2547, 2014.

[14] C.-C. Wu, H.-W. Su, C.-C. Lee, M.-J. Tang, and F.-C. Su, "Quantitative measurement of changes in adhesion force involving focal adhesion kinase during cell attachment, spread, and migration," Biochemical and Biophysical Research Communications, vol. 329, no. 1, pp. 256-265, 2005.

[15] K. A. Athanasiou, B. S. Thoma, D. R. Lanctot, D. Shin, C. M. Agrawal, and R. G. Lebaron, "Development of the cytodetachment technique to quantify mechanical adhesiveness of the single cell," Biomaterials, vol. 20, no. 23-24, pp. 2405-2415, 1999.

[16] J. Sanchez-Adams and K. A. Athanasiou, "Biomechanical characterization of single chondrocytes," in Cellular and Biomolecular Mechanics and Mechanobiology, vol. 4, pp. 247-266, Springer, Berlin, Germany, 2011.

[17] Y. Shen, M. R. Ahmad, M. Nakajima, S. Kojima, M. Homma, and T. Fukuda, "Evaluation of the single yeast cell's adhesion to ITO substrates with various surface energies via ESEM nanorobotic manipulation system," IEEE Transactions on Nanobioscience, vol. 10, no. 4, pp. 217-224, 2011.

[18] N. S. Tambe and B. Bhushan, "Scale dependence of micro/nanofriction and adhesion of MEMS/NEMS materials, coatings and lubricants," Nanotechnology, vol. 15, no. 11, pp. 1561-1570, 2004.

[19] Y. Shen, M. Nakajima, M. R. Ahmad, S. Kojima, M. Homma, and T. Fukuda, "Effect of ambient humidity on the strength of the adhesion force of single yeast cell inside environmentalSEM," Ultramicroscopy, vol. 111, no. 8, pp. 1176-1183, 2011.
[20] A. Hultgren, M. Tanase, C. S. Chen, G. J. Meyer, and D. H. Reich, "Cell manipulation using magnetic nanowires," Journal of Applied Physics, vol. 93, no. 10, pp. 7554-7556, 2003.

[21] N. Chronis and L. P. Lee, "Electrothermally activated SU-8 microgripper for single cell manipulation in solution," Journal of Microelectromechanical Systems, vol. 14, no. 4, pp. 857-863, 2005.

[22] E. W. H. Jager, O. Inganäs, and I. Lundström, "Microrobots for micrometer-size objects in aqueous media: potential tools for single-cell manipulation," Science, vol. 288, no. 5475, pp. 23352338, 2000. 

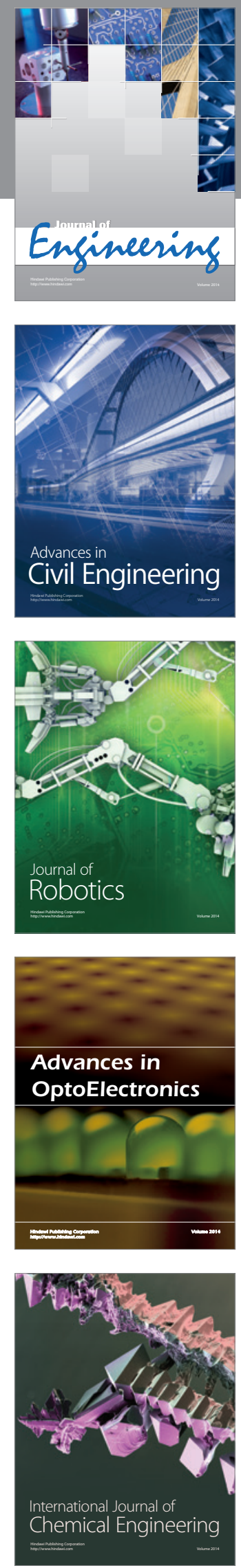

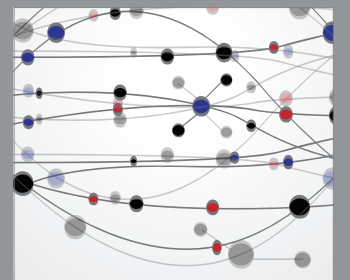

The Scientific World Journal
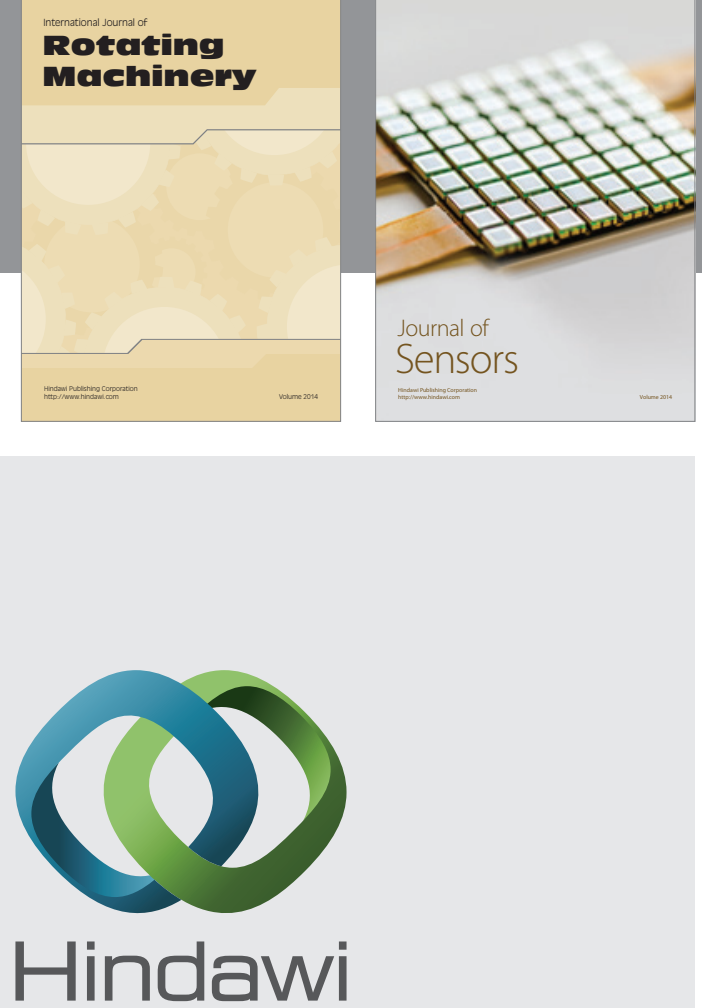

Submit your manuscripts at http://www.hindawi.com
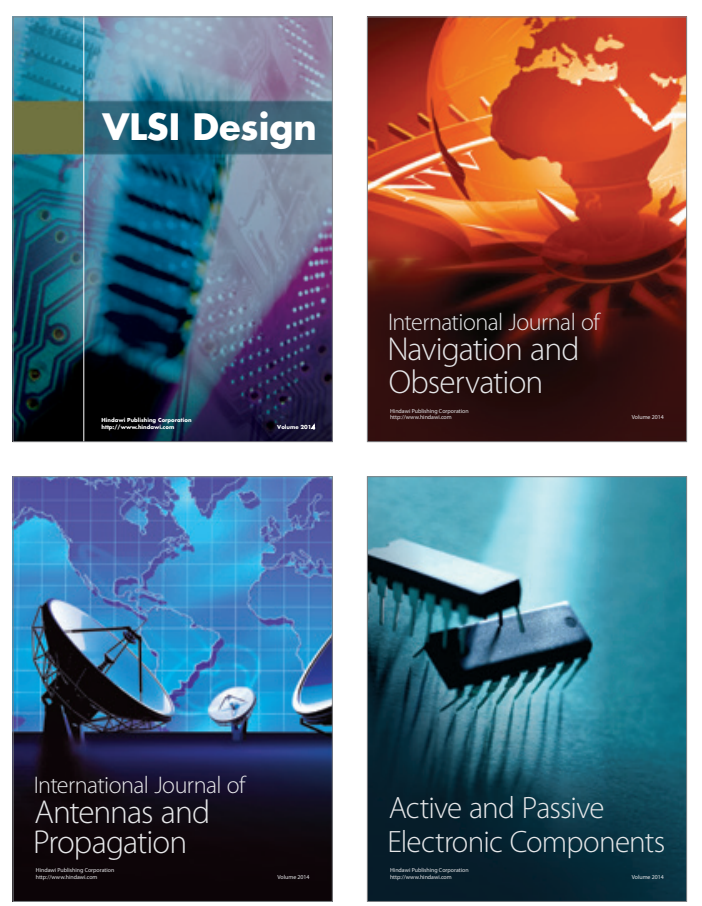
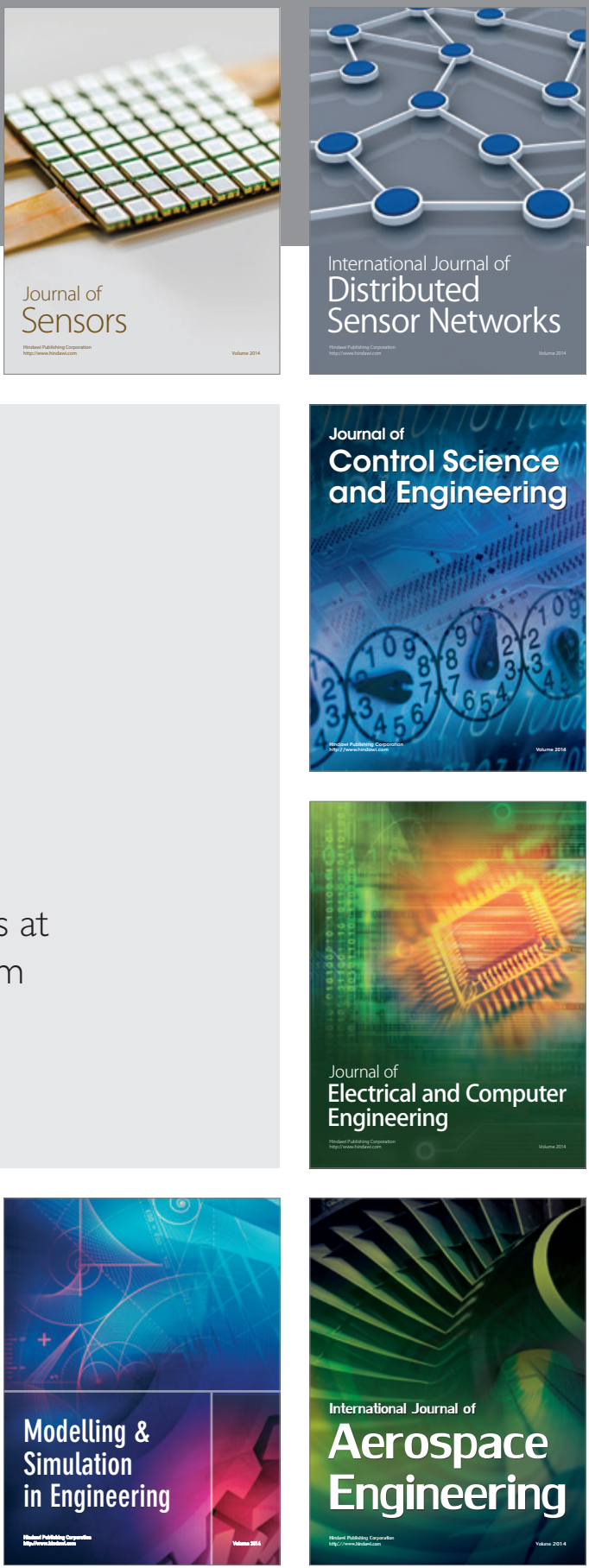

Journal of

Control Science

and Engineering
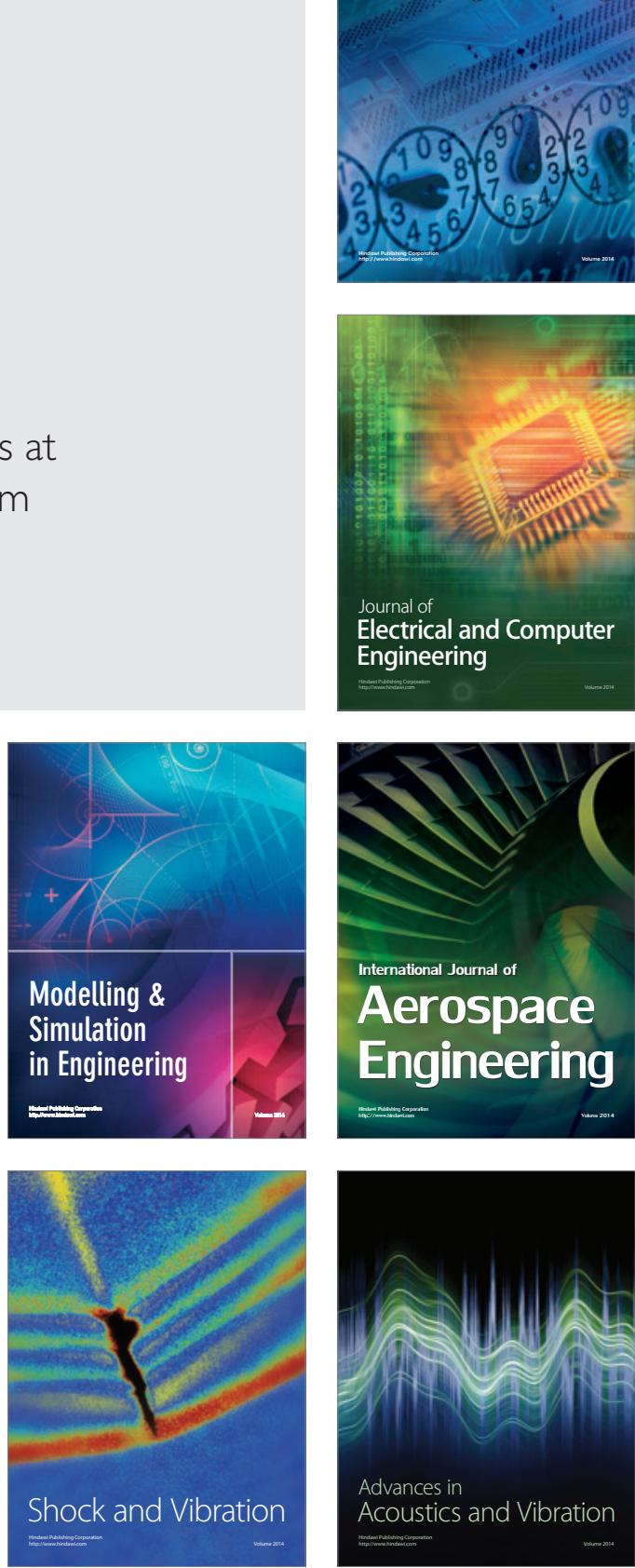